\section{(C) OPEN ACCESS}

\title{
Early work-related physical exposures and low back pain in midlife: the Cardiovascular Risk in Young Finns Study
}

\author{
T Lallukka, ${ }^{1,2}$ E Viikari-Juntura, ${ }^{1}$ J Viikari, ${ }^{3,4}$ M Kähönen, ${ }^{5,6}$ T Lehtimäki, ${ }^{7,8}$ \\ OT Raitakari, ${ }^{9,10}$ S Solovieva ${ }^{1}$
}

- Additional material is published online only. To view please visit the journal online (http://dx.doi.org/10.1136/ oemed-2016-103727)

For numbered affiliations see end of article.

\section{Correspondence to}

Dr Tea Lallukka, Finnish Institute of Occupational Health, P.O. Box 40, Helsinki FIN-00251, Finland: tea.lallukka@ttl.fi

TL, SS and EV-J contributed equally.

Received 24 March 2016 Revised 7 July 2016 Accepted 24 July 2016 Published Online First 11 August 2016
CrossMark

To cite: Lallukka T, Viikari-Juntura E, Viikari J, et al. Occup Environ Med 2017:74:163-168.

\section{ABSTRACT}

Objective To examine whether heavy physical workload in young adulthood increases the risk of local and radiating low back pain (LBP) in midlife.

Methods Longitudinal nationally representative Young Finns Study data among women $(n=414)$ and men $(n=324)$, aged 18-24 years in 1986 (baseline), were used. Physical heaviness of work was reported at baseline and follow-up (2007), and local and radiating LBP at follow-up. Covariates were age, smoking and body mass index. Logistic regression was used to examine the associations between physical heaviness of work and LBP. Additionally, the mediating effect of back pain at baseline was examined (the Sobel test).

Results After adjustment for the covariates, and as compared with sedentary/light physical workload, heavy physical workload was associated with radiating LBP among women (OR 4.09,95\% Cl 1.62 to 10.31) and men (OR 2.01, 95\% Cl 1.06 to 3.82). Among men, early back pain mediated the association ( $p$ value from the Sobel test=0.006). Among women, early exposure to physically heavy work showed the most consistent associations, while early and late exposures were associated with radiating and local LBP among men. Persistently heavy physical work was associated with radiating LBP among women and men.

Conclusions Physically heavy work at a young age can have a long-lasting effect on the risk of LBP, radiating LBP in particular. These results highlight the need to consider early and persistent exposures to prevent the adverse consequences of physical workload for the low back

Low back pain (LBP) is a highly prevalent and persistent condition and its determinants have been widely examined for decades. ${ }^{1}{ }^{2}$ Work-related factors are of particular interest, as acute and chronic pain among employees increase the risk of sickness absence and disability retirement. ${ }^{3}$ Globally, LBP attributable to occupation has a notable contribution to the number of disability-adjusted life years lost each year. ${ }^{4}$

Several physical workload factors, such as nonneutral trunk postures and various manual handling activities have been associated with $\mathrm{LBP}^{5} \mathrm{~A}$ recent individual participant data meta-analysis showed that the risk estimates for posture and force-related variables were small to moderate. ${ }^{6}$ Also, heavy physical work in general has been associated with LBP. $^{7}$ Physically heavy work usually involves

\section{What this paper adds}

- Physical workload is associated with back problems; however, the contribution of exposure to physically heavy work in young adulthood to the risk of low back pain (LBP) in midlife is poorly understood.

- This study showed that early exposure to heavy physical workload is associated with a high risk of radiating LBP after a follow-up of 21 years.

- The risks were particularly pronounced and robust among women, while among men, early back pain mediated the associations.

- In order to prevent LBP, care should be taken when young people enter into the labour market and are placed in jobs involving heavy physical work.

forceful activities and awkward postures that are loading for the low back.

The importance of life course perspective in the study of LBP has been highlighted. ${ }^{8-10}$ Although there are several studies on the associations between physical exposures and LBP, the focus has largely been on non-specific pain among midlife or older employees. A cross-sectional study showed that work-related physical factors are associated with LBP already in the first job. ${ }^{11}$ Similarly, in a Finnish study ${ }^{12}$ among individuals aged 16 years, physical workload was associated with incident LBP at the age of 18. However, the long-term consequences of early exposure to heavy physical work for the low back are largely unknown. Furthermore, the contribution of changes in physical workload during work careers to LBP has not been studied.

If the emergence of LBP outcomes in midlife has its roots in young adulthood, prevention needs to focus on early detection and modification of such risk factors. Therefore, we aimed to assess physical heaviness of work in early adulthood as a determinant of local and radiating LBP in midlife taking into consideration earlier back symptoms and persistency of workload over a period of more than two decades.

\section{METHODS}

Data

Data from the nationally representative Young Finns Study were used. ${ }^{13}$ The data have been 
repeatedly collected among participants who were 3, 6, 9, 12, 15 or 18 years at baseline in 1980 . In this study, the study base consisted of all those who were 18-24 years at the second follow-up in $1986(n=1265)$, and responded to the follow-up survey in $2007(\mathrm{n}=943,75 \%)$. More men than women were lost during the follow-up ( $28 \%$ vs $23 \%)$. There were no differences in age, body mass index (BMI), smoking, physical heaviness of work or LBP at baseline between participants and non-participants at follow-up.

Of 943 participants, 134 did not work either in 1986 or 2007 and were not eligible, leaving 809 participants in the study population. A total of 67 participants had missing information about physical heaviness of work in 1986, and 64 participants in 2007. Regarding the outcome, altogether six participants had missing data (radiating LBP in $2007(n=3)$, local LBP in $2007(n=6)$ and back pain in $1986(n=2))$. Altogether, 71 participants $(9 \%)$ had missing data on physical heaviness of work or a LBP variable either at baseline or follow-up, or both, leaving 738 participants (414 women and 324 men) in the analysis.

\section{Physical heaviness of work}

Physical heaviness of work was enquired in 1986 (baseline for this study) and 2007 (follow-up for this study) with a single question (how heavy is your work physically) using six response alternatives: (1) light sedentary work, (2) other sedentary work, (3) physically light work, involving standing and moving, (4) medium heavy work involving moving, (5) physically heavy work and (6) physically very heavy work. The responses were classified into three groups: sedentary/physically light work, medium heavy work and heavy physical work. Those with sedentary or physically light work served as the reference group. The assessment of occupational physical activity with a single question has been widely used in epidemiological studies andwhen categorised into three levels-has shown a high agreement with a detailed assessment of time spent in occupational physical activity. ${ }^{14}$

In addition, we examined cumulative exposure to physical workload by forming a new variable based on exposure to physically heavy work reported at baseline and follow-up: (1) those with all the time sedentary or physically light work, (2) early sedentary or physically light work; late at least medium heavy work, (3) early at least medium heavy work; late sedentary or physically light work and (4) all the time at least medium heavy work.

Moreover, we had information of the current occupation at baseline (Classification of Occupations 1980, Statistics Finland) and follow-up (Classification of Occupations 2001, Statistics Finland) and the type of work (eg, salaried employee, selfemployed, other). The distribution of the occupational characteristics of the study participants is shown in online supplementary appendix table 1 .

\section{Low back pain}

Outcomes at follow-up in 2007 were radiating LBP (pain radiating below the knee) and local LBP lasting $>7$ days during the preceding 12 months. ${ }^{15}$

In addition, the frequency of any back pain at baseline in 1986 was asked. The response alternatives were never or seldom, once a month, once a week and daily back pain. Those who reported that they seldom or never had back pain served as the reference category, while other responses were combined to indicate early back pain. No differentiation was made between local and radiating pain at baseline.

\section{Covariates}

Based on earlier evidence, ${ }^{15} 16$ we included as covariates age, ever-smoking and BMI (measured weight $/$ height $^{2}$ ) in 1986 . The associations between leisure-time physical activity and LBP are complex, and leisure-time physical activity is unlikely related to the earlier physical work exposures. Therefore, we did not adjust for leisure-time physical activity.

\section{Ethical approval}

The study has received ethical approvals from the local ethics committees.

\section{Statistical analyses}

Logistic regression analysis was used to examine the associations between physical heaviness of work and subsequent radiating and local LBP (ORs and their 95\% CIs). Crude associations are reported in model 0 , followed by adjustment for sex and age in model 1. Model 2 included sex, age, smoking and BMI. Model 3 included all the covariates, as well as back pain at baseline. To evaluate a mediating role of back pain at baseline in the longitudinal association between physical heaviness of work and LBP, we followed the Baron and Kenny process, ${ }^{17}$ which uses four steps of multiple regression model to establish the mediation. First, we estimated the effect of physical heaviness of work at baseline on LBP at follow-up. Second, we estimated the effect of back pain at baseline on LBP at follow-up. Third, we estimated the effect of physical heaviness of work at baseline on back pain at baseline. Fourth, we estimated the effect of physical heaviness of work at baseline with inclusion of back pain at baseline into the model. The significance of mediating effect was examined using the Sobel test. ${ }^{18}$ Owing to different risk factors, and difference in the prevalence of the determinant among women and men (table 1), all analyses were stratified by sex.

In order to assess the effect of missing data of self-reported physical heaviness of work on our results, we carried out a sensitivity analysis. First, using participants with complete information, we looked at the distribution of the physical heaviness of work by sex and occupation. Second, we used this occupation and sex-specific information to indicate physical heaviness of work for the 70 participants without information of physical heaviness of work (but with information of occupation). We were then able to analyse the results for 801 participants, leaving out only 8 participants $(1.0 \%)$ of our study population of 809 persons.

\section{RESULTS}

At follow-up, radiating LBP was reported by $20.1 \%$ of women and $19.4 \%$ of men, and local LBP by $36.2 \%$ of women and $34.0 \%$ of men (table 1). However, a larger proportion of men (29.3\%) than women $(5.1 \%)$ had been exposed to heavy physical work at baseline. Cumulative exposure to heavy physical work was also more common among men than women, as nearly a third of men and about a tenth of women had at least medium heavy work at baseline and follow-up.

Among women, medium heavy physical work (OR 2.35; 95\% CI 1.30 to 4.26 ) and heavy physical work (OR 3.86; 95\% CI 1.55 to 9.63 ) at baseline were associated with radiating LBP at follow-up (model 0, table 2). The associations slightly strengthened after adjusting for age (model 1), and remained statistically significant after adjusting for smoking and BMI (model 2). Back pain at baseline did not mediate the effect of physical workload on radiating LBP $(\mathrm{p}=0.34)$. 
Table 1 Distribution of key variables among women and men at baseline in 1986 and at follow-up in 2007

\begin{tabular}{|c|c|c|c|c|c|c|}
\hline & \multicolumn{2}{|c|}{ Women $(n=414)$} & \multicolumn{2}{|c|}{ Men $(n=324)$} & \multicolumn{2}{|c|}{ All $(n=738)$} \\
\hline & $\mathbf{N}$ & Per cent & $\mathrm{N}$ & Per cent & $\mathrm{N}$ & Per cent \\
\hline \multicolumn{7}{|l|}{ Baseline (1986) } \\
\hline \multicolumn{7}{|l|}{ Age (years) } \\
\hline 18 & 159 & 38.4 & 111 & 34.3 & 270 & 36.6 \\
\hline 21 & 134 & 32.4 & 114 & 35.2 & 248 & 33.6 \\
\hline 24 & 121 & 29.2 & 99 & 30.6 & 220 & 29.8 \\
\hline Ever smoking & 155 & 37.4 & 143 & 44.1 & 298 & 40.4 \\
\hline Back pain & 161 & 38.9 & 110 & 34.0 & 271 & 36.7 \\
\hline Mean (SD) body mass index $\left(\mathrm{kg} / \mathrm{m}^{2}\right)$ & \multicolumn{2}{|c|}{21.7 (SD 2.7) } & \multicolumn{2}{|c|}{22.5 (SD 2.8) } & \multicolumn{2}{|c|}{22.0 (SD 2.8) } \\
\hline \multicolumn{7}{|l|}{ Physical workload } \\
\hline Sedentary or physically light work & 326 & 78.7 & 169 & 52.2 & 495 & 67.1 \\
\hline Medium heavy work & 67 & 16.2 & 60 & 18.5 & 127 & 17.2 \\
\hline Heavy work & 21 & 5.1 & 95 & 29.3 & 116 & 15.7 \\
\hline \multicolumn{7}{|l|}{ Follow-up (2007) } \\
\hline \multicolumn{7}{|l|}{ Physical workload } \\
\hline Sedentary or physically light work & 296 & 71.5 & 183 & 56.5 & 479 & 64.9 \\
\hline Medium heavy work & 90 & 21.7 & 85 & 26.2 & 175 & 23.7 \\
\hline Heavy work & 28 & 6.8 & 56 & 17.3 & 84 & 11.4 \\
\hline Radiating low back pain & 83 & 20.1 & 63 & 19.4 & 146 & 19.8 \\
\hline Local low back pain & 150 & 36.2 & 110 & 34.0 & 260 & 35.2 \\
\hline \multicolumn{7}{|l|}{ Cumulative physical workload 1986-2007 } \\
\hline All the time sedentary or physically light work & 252 & 60.9 & 124 & 38.3 & 376 & 51.0 \\
\hline $\begin{array}{l}\text { Early sedentary or physically light work; late at } \\
\text { least medium heavy work }\end{array}$ & 74 & 17.9 & 45 & 13.9 & 119 & 16.1 \\
\hline $\begin{array}{l}\text { Early at least medium heavy work; late sedentary or } \\
\text { physically light work }\end{array}$ & 44 & 10.6 & 59 & 18.2 & 103 & 14.0 \\
\hline All the time at least medium heavy work & 44 & 10.6 & 96 & 29.6 & 140 & 19.0 \\
\hline
\end{tabular}

Table 2 Associations of physical heaviness of work at baseline in 1986 with radiating and local low back pain at follow-up in 2007 (ORs and their $95 \%$ Cls)

\begin{tabular}{|c|c|c|c|c|c|c|c|c|}
\hline \multirow[b]{2}{*}{ Physical heaviness of work at baseline } & \multicolumn{2}{|c|}{ Model 0: crude } & \multicolumn{2}{|c|}{$\begin{array}{l}\text { Model 1: adjusted for } \\
\text { age }\end{array}$} & \multicolumn{2}{|c|}{$\begin{array}{l}\text { Model 2: adjusted for } \\
\text { age, smoking and BMI }\end{array}$} & \multicolumn{2}{|c|}{$\begin{array}{l}\text { Model 3: adjusted for } \\
\text { age, smoking and BMI, } \\
\text { and mediating effects } \\
\text { of pain in } 1986\end{array}$} \\
\hline & OR & $95 \% \mathrm{Cl}$ & OR & $95 \% \mathrm{Cl}$ & OR & $95 \% \mathrm{Cl}$ & OR & $95 \% \mathrm{Cl}$ \\
\hline \multicolumn{9}{|l|}{ Radiating low back pain } \\
\hline \multicolumn{9}{|l|}{ Women $(n=414)$} \\
\hline Sedentary or physically light work & 1.00 & & 1.00 & & 1.00 & & 1.00 & \\
\hline Medium heavy work & 2.35 & (1.30 to 4.26$)$ & 2.55 & (1.37 to 4.71$)$ & 2.41 & (1.29 to 4.50$)$ & 2.43 & (1.30 to 4.54$)$ \\
\hline Heavy work & 3.86 & (1.55 to 9.63$)$ & 4.06 & (1.62 to 10.18$)$ & 4.09 & (1.62 to 10.31$)$ & 3.98 & (1.57 to 10.07$)$ \\
\hline \multicolumn{9}{|l|}{ Men $(n=324)$} \\
\hline Sedentary or physically light work & 1.00 & & 1.00 & & 1.00 & & 1.00 & \\
\hline Medium heavy work & 1.84 & (0.88 to 3.85$)$ & 1.74 & (0.83 to 3.65$)$ & 1.68 & (0.79 to 3.59$)$ & 1.23 & (0.56 to 2.72$)$ \\
\hline Heavy work & 2.16 & (1.15 to 4.05$)$ & 2.06 & (1.09 to 3.88$)$ & 2.01 & (1.06 to 3.82$)$ & 1.56 & (0.80 to 3.07$)$ \\
\hline \multicolumn{9}{|l|}{ Local low back pain } \\
\hline \multicolumn{9}{|l|}{ Women $(n=414)$} \\
\hline Sedentary or physically light work & 1.00 & & 1.00 & & 1.00 & & 1.00 & \\
\hline Medium heavy work & 1.39 & (0.81 to 2.38$)$ & 1.34 & (0.77 to 2.32$)$ & 1.26 & (0.72 to 2.20$)$ & 1.27 & (0.72 to 2.22$)$ \\
\hline Heavy work & 2.13 & (0.88 to 5.17$)$ & 2.08 & (0.86 to 5.07$)$ & 2.11 & (0.86 to 5.15$)$ & 2.03 & (0.82 to 4.98$)$ \\
\hline \multicolumn{9}{|l|}{ Men $(n=324)$} \\
\hline Sedentary or physically light work & 1.00 & & 1.00 & & 1.00 & & 1.00 & \\
\hline Medium heavy work & 1.99 & (1.08 to 3.66$)$ & 2.14 & (1.15 to 3.99$)$ & 2.06 & (1.09 to 3.89 ) & 1.82 & (0.95 to 3.48$)$ \\
\hline Heavy work & 1.66 & (0.97 to 2.82$)$ & 1.77 & (1.03 to 3.03$)$ & 1.71 & (0.99 to 2.95$)$ & 1.55 & (0.89 to 2.71$)$ \\
\hline
\end{tabular}


Regarding local LBP, none of the associations was statistically significant.

Among men, only heavy physical work (OR 2.16; 95\% CI 1.15 to 4.05 ) was associated with subsequent radiating LBP (model 0 ). After adjusting for age (model 1), the associations slightly attenuated and remained practically unaffected after further adjusting for smoking and BMI (model 2). In contrast to women, early exposure to physical workload had direct and indirect (via back pain at baseline) effect on radiating ( $p$ value for mediation 0.006) and local LBP ( $p$ value for mediation 0.03 ) at follow-up.

Next, to confirm the association between early exposure to heavy physical workload and subsequent LBP in midlife, we repeated the analyses among only those who were pain-free at baseline (table 3). Among women, the associations for radiating LBP remained. Among men, the magnitude of the associations was similar, but the associations were not statistically significant. Correspondingly, associations were similar for local LBP among women and men.

Finally, we examined the associations of cumulative exposure to heavy physical work at baseline and follow-up with radiating and local LBP (table 4). Among women, early at least medium heavy work was associated with radiating LBP, even if the workload had decreased to sedentary or physically light (OR 2.71; 95\% CI 1.32 to 5.59 )(model 0 ). A higher risk of radiating LBP was found also for those with repeated exposure to at least medium heavy work (OR 3.32; 95\% CI 1.64 to 6.73). Both of these associations slightly strengthened after adjusting for age (model 1) and remained after considering smoking and BMI (model 2).

Among men, early (OR 4.17; 95\% CI 1.71 to 10.20 ) and later (OR 4.51; 95\% CI 1.97 to 10.35 ) exposure to at least medium heavy work were associated with radiating LBP. Further adjustments had a negligible effect on the association.

Cumulative exposure to heavy physical workload was associated with also local LBP among women and men. Among women, the association was statistically significant only regarding a change from an early sedentary or physically light work to later at least medium heavy work. However, among men, all those with early, late or both types of exposure to heavy physical work were at a higher risk for local LBP at follow-up. The associations remained after all adjustments.

\section{Sensitivity analyses}

As a sensitivity analysis, we carried out our main analyses including participants with missing questionnaire data on the exposure of interest. For baseline exposures, the point estimates of the low back outcomes were in general lower and the CIs narrower (see online supplementary appendix table 2). The association between physical heaviness of work at baseline and radiating LBP at follow-up was no longer statistically significant among men. For cumulative exposures the values of the point estimates did not change markedly; however, their precision increased (see online supplementary appendix table 3 ).

\section{DISCUSSION}

With a nationally representative sample of Finnish women and men, we found that exposure to heavy physical work at the age of 18-24 was associated with radiating LBP after a follow-up of more than two decades. The association was particularly pronounced among women. Among men, the effect was largely mediated by early back pain. Early physical workload was associated with local LBP among men only. Furthermore, among men, early and late heavy physical work increased the risk of local and radiating LBP, while among women early exposures dominated as a risk factor for radiating LBP in midlife.

\section{Interpretation}

There is a lack of comparable studies focusing on the role of physical heaviness of work during the earlier career in the aetiology of LBP. Physically heavy work was associated with LBP

Table 3 Associations of physical heaviness of work at baseline in 1986 with radiating and local low back pain at follow-up in 2007 (ORs and their $95 \%$ Cls) among participants who were pain-free in 1986

\begin{tabular}{|c|c|c|c|c|c|c|}
\hline \multirow[b]{2}{*}{ Physical heaviness of work at baseline } & \multicolumn{2}{|c|}{ Model 0: crude } & \multicolumn{2}{|c|}{ Model 1: adjusted for age } & \multicolumn{2}{|c|}{$\begin{array}{l}\text { Model 2: adjusted for age, } \\
\text { smoking and BMI }\end{array}$} \\
\hline & OR & $95 \% \mathrm{Cl}$ & OR & $95 \% \mathrm{Cl}$ & OR & $95 \% \mathrm{Cl}$ \\
\hline \multicolumn{7}{|l|}{ Radiating low back pain } \\
\hline \multicolumn{7}{|l|}{ Women $(n=253)$} \\
\hline Sedentary or physically light work & 1.00 & & 1.00 & & 1.00 & \\
\hline Medium heavy work & 3.65 & (1.69 to 7.88$)$ & 4.08 & (1.83 to 9.11$)$ & 3.94 & (1.72 to 9.02$)$ \\
\hline Heavy work & 5.87 & (1.67 to 20.65 ) & 6.59 & (1.83 to 23.81 ) & 6.22 & (1.70 to 22.77$)$ \\
\hline \multicolumn{7}{|l|}{ Men $(n=214)$} \\
\hline Sedentary or physically light work & 1.00 & & 1.00 & & 1.00 & \\
\hline Medium heavy work & 1.98 & (0.64 to 6.13$)$ & 1.83 & (0.59 to 5.7 ) & 1.98 & (0.61 to 6.41$)$ \\
\hline Heavy work & 2.03 & (0.80 to 5.15$)$ & 2.04 & (0.80 to 5.2$)$ & 2.17 & (0.84 to 5.62$)$ \\
\hline \multicolumn{7}{|l|}{ Local low back pain } \\
\hline \multicolumn{7}{|l|}{ Women $(n=253)$} \\
\hline Sedentary or physically light work & 1.00 & & 1.00 & & 1.00 & \\
\hline Medium heavy work & 1.58 & (0.79 to 3.17$)$ & 1.58 & (0.77 to 3.21$)$ & 1.39 & (0.67 to 2.91$)$ \\
\hline Heavy work & 2.06 & (0.60 to 7.00$)$ & 2.05 & (0.60 to 7.05$)$ & 1.87 & (0.53 to 6.66$)$ \\
\hline \multicolumn{7}{|l|}{ Men $(n=214)$} \\
\hline Sedentary or physically light work & 1.00 & & 1.00 & & 1.00 & \\
\hline Medium heavy work & 1.79 & (0.77 to 4.16$)$ & 1.97 & (0.84 to 4.65$)$ & 2.07 & (0.86 to 4.99$)$ \\
\hline Heavy work & 1.59 & (0.80 to 3.19$)$ & 1.62 & (0.81 to 3.27$)$ & 1.66 & (0.82 to 3.38$)$ \\
\hline
\end{tabular}


Table 4 Associations of cumulative exposure to heavy physical work at baseline (1986) and follow-up (2007) with radiating and local low back pain at follow-up (ORs and their 95\% Cls)

\begin{tabular}{|c|c|c|c|c|c|c|c|c|}
\hline & \multicolumn{2}{|c|}{$\begin{array}{l}\text { At least medium heavy } \\
\text { workload }\end{array}$} & \multicolumn{2}{|c|}{ Model 0: crude } & \multicolumn{2}{|c|}{ Model 1: adjusted for age } & \multicolumn{2}{|c|}{$\begin{array}{l}\text { Model 2: adjusted for } \\
\text { age, smoking and BMI }\end{array}$} \\
\hline & At baseline & At follow-up & OR & $95 \% \mathrm{Cl}$ & OR & $95 \% \mathrm{Cl}$ & OR & $95 \% \mathrm{Cl}$ \\
\hline \multicolumn{9}{|c|}{ Radiating low back pain } \\
\hline \multirow[t]{4}{*}{ Women $(n=414)$} & - & - & 1.00 & & 1.00 & & 1.00 & \\
\hline & + & - & 2.71 & (1.32 to 5.59 ) & 2.86 & (1.37 to 5.95$)$ & 2.76 & (1.32 to 5.77$)$ \\
\hline & - & + & 1.60 & (0.83 to 3.08 ) & 1.55 & (0.80 to 2.99 ) & 1.52 & (0.78 to 2.95 ) \\
\hline & + & + & 3.32 & (1.64 to 6.73$)$ & 3.55 & (1.72 to 7.31$)$ & 3.42 & (1.65 to 7.07 ) \\
\hline \multirow[t]{4}{*}{ Men $(n=324)$} & - & - & 1.00 & & 1.00 & & 1.00 & \\
\hline & + & - & 4.51 & (1.97 to 10.35$)$ & 4.29 & (1.86 to 9.87 ) & 4.32 & (1.84 to 10.12$)$ \\
\hline & - & + & 4.17 & (1.71 to 10.2 ) & 4.37 & (1.77 to 10.75 ) & 4.46 & (1.80 to 11.08$)$ \\
\hline & + & + & 2.88 & (1.31 to 6.31$)$ & 2.76 & (1.26 to 6.08$)$ & 2.75 & (1.23 to 6.11$)$ \\
\hline \multicolumn{9}{|c|}{ Local low back pain } \\
\hline \multirow[t]{4}{*}{ Women $(n=414)$} & - & - & 1.00 & & 1.00 & & 1.00 & \\
\hline & + & - & 1.70 & (0.88 to 3.26 ) & 1.64 & (0.85 to 3.16$)$ & 1.56 & (0.80 to 3.03$)$ \\
\hline & - & + & 1.80 & (1.06 to 3.05 ) & 1.84 & (1.08 to 3.15$)$ & 1.79 & (1.04 to 3.06$)$ \\
\hline & + & + & 1.86 & (0.97 to 3.56$)$ & 1.78 & (0.92 to 3.44$)$ & 1.71 & (0.88 to 3.31$)$ \\
\hline \multirow[t]{4}{*}{ Men $(n=324)$} & - & - & 1.00 & & 1.00 & & 1.00 & \\
\hline & + & - & 2.76 & (1.43 to 5.35$)$ & 2.98 & (1.53 to 5.82 ) & 2.95 & (1.49 to 5.84 ) \\
\hline & - & + & 2.18 & (1.06 to 4.52 ) & 2.14 & (1.03 to 4.44$)$ & 2.18 & (1.04 to 4.56$)$ \\
\hline & + & + & 1.97 & (1.09 to 3.53 ) & 2.07 & (1.15 to 3.75$)$ & 2.01 & (1.10 to 3.67$)$ \\
\hline
\end{tabular}

2 years later in the Northern Finland Birth Cohort and with LBP after 1 year in newly employed young Belgians. ${ }^{19}$ Furthermore, in agreement with findings from the Northern Finland Birth Cohort, we observed sex differences in the prevalence of early physical work exposures. ${ }^{12}$ None of the previous studies distinguished between radiating and local LBP. Moreover, as we focused on general heaviness of work and not on the effects of local loads on the low back, our results cannot be directly compared with the results of previous reviews on different types of local physical exposures and LBP. 5616

There is very little evidence of the cumulative effects of physical workloads on LBP outcomes in midlife. In a cross-sectional study among Finns aged 30-64 years, retrospectively enquired physical demands of work during life course were associated with sciatica only among men. ${ }^{20}$ In women, such demands were associated with sciatica among only those who were not working, suggesting health-based selection out of the labour market.

The current results highlight the effects of persistently heavier physical workload during working life on the risk of local and radiating LBP among women and men. Moreover, among women, early exposure to at least medium heavy workload appears to be more important, while among men either early or late medium heavy or heavy physical workload can have adverse effects on the low back. These findings emphasise the public health and societal relevance of prevention and modification of early risk factors particularly among women.

In all, sex differences found by us and the earlier studies ${ }^{4}$ confirm that women and men should be examined separately when focusing on risk factors of LBP. Some explanations for the found sex differences can be suggested. First, parental socioeconomic position could be assumed to partly account for the differences. Some men could have had physical exposures already in childhood, and could thereby have heavier physical work in young adulthood jointly contributing to their risk of LBP outcomes in midlife. Accordingly, being raised in a farm has been associated with low back disorders among men, but not among women. ${ }^{15}$ Second, heavy physical work was more prevalent among men as compared with women, which is likely due to jobs being gender segregated. Men could also enter the work life earlier, and be more likely than women to continue in heavy physical work.

A review of systematic reviews, attempting to assess causality against Hill's criteria, concluded that a causal association has not been established for any of the included exposures. ${ }^{5}$ However, the results of our longitudinal study suggest that among women, the robust association between heavy physical workload and radiating LBP could be causal and direct, while in men the effect of early exposure to heavy physical work might be indirectly causal (mediated by earlier back pain). There is also a biologically plausible explanation for the association via mechanical loading. ${ }^{21}$

Overall, exposure to heavy physical workload in young adulthood increases the risk of low back problems, especially radiating LBP even after decades. Since many adults are continuously exposed to physically demanding work, and musculoskeletal diseases remain a key cause of work disability, ${ }^{22}$ health and societal significance of the association between early physical exposures and subsequent LBP are notable.

\section{Methodological considerations}

Some limitations and strengths of our study need to be acknowledged. The assessment of physical heaviness of work was based on a single question. However, such questions have been widely used in epidemiological studies and have shown high validity. ${ }^{1423}$ About $8 \%$ of the participants had missing data regarding self-reported physical heaviness of work. Our sensitivity analyses suggest that exclusion of them from the main analyses had negligible effects on our results. The relatively small sample size led to an overestimation of ORs and reduced precision of the estimates, indicated by the wide CIs. However, it is unlikely that our non-significant findings would turn to be significant with a larger sample size.

Our long follow-up could be seen as a limitation and strength. On the one hand, we cannot rule out that there could be changes over time in physical exposures or unmeasured 
confounding. On the other hand, the 21-year follow-up enabled us to examine long-term consequences of early exposure to physical workload. With regard to cumulative exposure to physical workload, it needs to be noted that health-related selection could have affected our results. Thus, those with the most severe back or other health problems at baseline might have exited the labour market over follow-up, or changed to lighter work. $^{24} 25$ If that were the case, the results could be conservative. Finally, examining those with sedentary or light exposures as a reference group could be questioned. Albeit the earlier evidence is not fully consistent, ${ }^{5}$ sedentary work appears not to increase the risk for LBP based on reviews and a recent Finnish study. ${ }^{20} 26 \quad 27$ Accordingly, we think that for our purpose of assessing the effects of medium heavy or heavy physical workload on LBP, those with sedentary work can serve as a comparison group.

Strengths of our prospective study comprise the use of rich, representative data with relatively little loss to follow-up, ${ }^{13}$ the inclusion of key covariates of $\mathrm{LBP}^{16}$ and the ability to examine separately women and men who have different jobs and thereby varying exposures which is likely to affect the risk of LBP. A further strength is that a distinction was made between local and radiating LBP.

In conclusion, early exposure to heavier physical workload may have a long-lasting effect on the risk of LBP, radiating LBP in particular. In the prevention of low back problems, timely prevention of early occupational exposures should not be neglected. Overall, this information can be used in the current efforts to extend work careers. Care should be taken when young people enter into the labour market and are placed in jobs involving heavy physical work.

\author{
Author affiliations \\ ${ }^{1}$ Finnish Institute of Occupational Health, Helsinki, Finland \\ ${ }^{2}$ Department of Public Health, University of Helsinki, Helsinki, Finland \\ ${ }^{3}$ Department of Medicine, University of Turku, Turku, Finland \\ ${ }^{4}$ Division of Medicine, Turku University Hospital, Turku, Finland \\ ${ }^{5}$ Department of Clinical Physiology, University of Tampere, Tampere, Finland \\ ${ }^{6}$ Tampere University Hospital, Tampere, Finland \\ ${ }^{7}$ Department of Clinical Chemistry, Fimlab Laboratories, Tampere, Finland \\ ${ }^{8}$ School of Medicine, University of Tampere, Tampere, Finland \\ ${ }^{9}$ Research Centre of Applied and Preventive Cardiovascular Medicine, University of \\ Turku, Turku, Finland \\ ${ }^{10}$ Department of Clinical Physiology and Nuclear Medicine, Turku University Hospital, \\ Turku, Finland
}

Contributors TL, EV-J and SS conceived and designed the study, and TL and SS analysed the data. TL is the guarantor of the study and prepared the first version of the manuscript. TL, EV-J and SS contributed equally to the final version. All other authors commented the manuscript and approved the submission of the final version.

Funding This study was supported by the Academy of Finland (grant 129364). Tea Lallukka is supported by the Academy of Finland (grant 287488). The Young Finns Study has been financially supported by the Academy of Finland: grants 286284 (T.L.), 134309 (Eye), 126925, 121584, 124282, 129378 (Salve), 117787 (Gendi) and 41071 (Skidi); the Social Insurance Institution of Finland; Kuopio, Tampere and Turku University Hospital Medical Funds (grant X51001 for T.L.); Juho Vainio Foundation; Paavo Nurmi Foundation; Finnish Foundation of Cardiovascular Research; Finnish Cultural Foundation; Tampere Tuberculosis Foundation; Emil Aaltonen Foundation; and Yrjö Jahnsson Foundation. The expert technical assistance in data management by Irina Lisinen and Ville Aalto is gratefully acknowledged.

Competing interests None declared.

Ethics approval The study has received ethical approvals from the local ethics committees.
Provenance and peer review Not commissioned; externally peer reviewed.

\section{REFERENCES}

1 Hoy D, Brooks $P$, Blyth $F$, et al. The Epidemiology of low back pain. Best Pract Res Clin Rheumatol 2010;24:769-81.

2 Taylor JB, Goode AP, George SZ, et al. Incidence and risk factors for first-time incident low back pain: a systematic review and meta-analysis. Spine $J$ 2014:14:2299-319.

3 Saastamoinen $\mathrm{P}$, Laaksonen $\mathrm{M}$, Kääriä $\mathrm{SM}$, et al. Pain and disability retirement: a prospective cohort study. Pain 2012;153:526-31.

4 Driscoll T, Jacklyn $\mathrm{G}$, Orchard J, et al. The global burden of occupationally related low back pain: estimates from the Global Burden of Disease 2010 study. Ann Rheum Dis 2014:73:975-81.

5 Kwon BK, Roffey DM, Bishop PB, et al. Systematic review: occupational physical activity and low back pain. Occup Med 2011;61:541-8.

6 Griffith LE, Shannon HS, Wells RP, et al. Individual participant data meta-analysis of mechanical workplace risk factors and low back pain. Am J Public Health 2012;102:309-18.

7 Solovieva S, Pehkonen I, Kausto J, et al. Development and validation of a job exposure matrix for physical risk factors in low back pain. PLOS ONE 2012;7: e48680.

8 Macfarlane GJ. Life-course influences on chronic pain in adults. In: Croft B, Blyth FM, van der Windt D, eds. Chronic Pain Epidemiology: From Aetiology to Public Health. New York: Oxford University Press, 2010:177-84.

9 Dunn KM. Extending conceptual frameworks: life course epidemiology for the study of back pain. BMC Musculoskelet Disord 2010;11:23.

10 Dunn KM, Hestbaek L, Cassidy JD. Low back pain across the life course. Best Pract Res Clin Rheumatol 2013;27:591-600.

11 Van Nieuwenhuyse A, Fatkhutdinova L, Verbeke G, et al. Risk factors for first-ever low back pain among workers in their first employment. Occup Med 2004;54:513-19.

12 Mikkonen P, Viikari-Juntura E, Remes J, et al. Physical workload and risk of low back pain in adolescence. Occup Environ Med 2012;69:284-90.

13 Raitakari OT, Juonala M, Rönnemaa T, et al. Cohort profile: the cardiovascular risk in Young Finns Study. Int J Epidemiol 2008;37:1220-6.

14 Yore MM, Bowles HR, Ainsworth BE, et al. Single versus multiple item questions on occupational physical activity. J Phys Act Health 2006;3:102-11.

15 Lallukka T, Viikari-Juntura E, Raitakari OT, et al. Childhood and adult socio-economic position and social mobility as determinants of low back pain outcomes. Eur J Pain 2014;18:128-38.

16 da Costa BR, Vieira ER. Risk factors for work-related musculoskeletal disorders: a systematic review of recent longitudinal studies. Am J Ind Med 2010;53:285-323.

17 Baron RM, Kenny DA. The moderator-mediator variable distinction in socia psychological research: conceptual, strategic, and statistical considerations. J Pers Soc Psychol 1986;51:1173-82.

18 Sobel ME. Asymptotic intervals for indirect effects in structural equations models. In: Leinhart S, ed. Sociological Methodology. San Francisco: Jossey-Bass, 1982:290-312.

19 Van Nieuwenhuyse A, Somville PR, Crombez G, et al. The role of physical workload and pain related fear in the development of low back pain in young workers: evidence from the BelCoBack Study; results after one year of follow up. Occup Environ Med 2006;63:45-52.

20 Kaila-Kangas L, Leino-Arjas P, Karppinen J, et al. History of physical work exposures and clinically diagnosed sciatica among working and nonworking Finns aged 30 to 64. Spine 2009;34:964-9.

21 Kumar S. Theories of musculoskeletal injury causation. Ergonomics 2001;44:17-47.

22 Finnish Centre of Pensions and The Social Insurance Institute of Finland. The Statistical Yearbook of Pensioners in Finland 2012. Helsinki: Finnish Centre for Pensions; The Social Insurance Institution of Finland, 2013:1-142.

23 Stock SR, Fernandes R, Delisle A, et al. Reproducibility and validity of workers' self-reports of physical work demands. Scand J Work Environ Health 2005;31:409-37.

$24 \mathrm{Li} C Y$, Sung FC. A review of the healthy worker effect in occupational epidemiology. Occup Med 1999;49:225-9.

25 Wilcosky T, Wing S. The healthy worker effect. Selection of workers and work forces. Scand J Work Environ Health 1987;13:70-2.

26 Roffey DM, Wai EK, Bishop P, et al. Causal assessment of occupational sitting and low back pain: results of a systematic review. Spine J 2010;10:252-61.

27 Bakker EWP, Verhagen AP, van Trijffel E, et al. Spinal mechanical load as a risk factor for low back pain: a systematic review of prospective cohort studies. Spine 2009;34:E281-93. 